\title{
Thermophoretic Deposition of Particles onto Cold Surfaces of Bodies in Two-Dimensional and Axisymmetric Flows ${ }^{1}$
}

\author{
C. SHEN \\ Institute of Mechanics, Chinese Academy of Sciences, Beijing, China, 100080
}

Received June 22, 1987; accepted January 26, 1988

\begin{abstract}
The problem of thermophoretic deposition of small particles onto cold surfaces is studied in twodimensional and axisymmetric flow fields. The particle concentration equation is solved numerically together with the momentum and energy equations in the laminar boundary layer with variable density effect included. It is shown explicitly to what extent the particle concentration and deposition rate at the wall are influenced by the density variation effect for external flow past bodies. The general numerical procedure is given for two-dimensional and axisymmetric cases and is illustrated with examples of thermophoretic deposition of particles in flows past a cold cylinder and a sphere. 1989 Academic Press, Inc.
\end{abstract}

\section{INTRODUCTION}

The problem of thermophoretic deposition of particles has attracted the attention of many investigators ( paper is to treat the external problem and to provide a general method of calculating the thermophoretic deposition rate of particles suspended in two-dimensional or axisymmetric flow past a body held at a lower temperature than that of the flow. Some of the aforementioned papers $(1,5,7)$ treated the problem of flow past a body, but only Goren (1) who investigated the flow past a flat plate took into account the density variation effect which is vital to the analysis of thermophoresis, as pointed out by Abbott (8). In this paper special consideration is given to the flow near the stagnation point of a cylinder and a sphere and past a flat plate to show explicitly how the particle concentration and deposition rate at the wall are influenced by the density variation effect in these flow systems. Then the general case of two-dimensional and axisymmetric flow past bodies of arbitrary shape is considered. A general numerical procedure is given and is illustrated by examples for flow past a cylinder and a sphere.

The formulation of the problem and the

\footnotetext{
${ }^{1}$ Supported by NSFC.
}

basic equations are given in Section 2. The solution in flows near the stagnation point on a cylinder and a sphere and past a flat plate is given in Section 3. The method of solution used for the general case is given in Section 4. Examples of the calculation of thermophoretic deposition for flow past a cylinder and a sphere are presented in Section 5 followed by a section of discussion.

\section{FORMULATION OF THE PROBLEM}

In this paper our consideration of thermophoretic deposition focuses on external flow problems in which a stream of gas containing suspended particles flows past a body held at a lower temperature than that of the oncoming stream. The particle size and concentration are assumed to be small so that the velocity and temperature fields of the gas can be determined as in a flow free of particles. For the range of Reynolds and Mach numbers usually encountered in thermophoretic problems the stream of gas can be assumed to flow around the body irrotationally and as an incompressible fluid outside the laminar boundary layer near the surface inside of which the variation of density must be allowed for. Compressibility is retained near the body despite the smallness of the velocity because the thermophoretic velocity is proportional to the relative variation 
of the temperature which in turn is proportional to the relative variation of the density for the ideal gas. For a two-dimensional or axisymmetric, laminar, compressible flow the mass conservation equation and the momentum and energy boundary layer equations in the boundary layer coordinate system can be written as

$$
\begin{array}{r}
\frac{\partial}{\partial x}\left(r^{k} \rho u\right)+\frac{\partial}{\partial y}\left(r^{k} \rho v\right)=0 \\
\rho u \frac{\partial u}{\partial x}+\rho v \frac{\partial u}{\partial y} \\
=-\frac{d p}{d x}+\frac{1}{r^{k}} \frac{\partial}{\partial y}\left[r^{k}\left(\mu \frac{\partial u}{\partial y}\right)\right] \\
\rho u \frac{\partial I}{\partial x}+\rho v \frac{\partial I}{\partial y} \\
=\frac{1}{r^{k}} \frac{\partial}{\partial y}\left[r^{k}\left\{\mu\left(1-\frac{1}{\sigma}\right) u \frac{\partial u}{\partial y}+\frac{\mu}{\sigma} \frac{\partial I}{\partial y}\right\}\right]
\end{array}
$$

where $x$ represents streamwise distance along the surface from the leading edge or the stagnation point, $y$ is the position coordinate normal to the boundary, $u$ and $v$ are the gas velocity components parallel and normal to the surface, $r$ is the distance of the considered point to the axis, $k=1$ for axisymmetric and $k=0$ for two-dimensional flows (see Fig. 1), $\mu$ is the viscosity of the gas, and $\sigma=\mu c_{\mathrm{p}} / k_{\mathrm{g}}$ is the ratio of the kinematic viscosity $\nu$ to the thermal diffusivity $k_{\mathrm{g}} / \rho c_{\mathrm{p}}$, is equal to 0.72 for air, and lies within the range 0.66 to 0.80 for common gas, $I=c_{\mathrm{p}} T+u^{2} / 2$. $^{2}$

The distribution of particle concentration in general is governed by convection, diffusion, and thermophoresis and is determined by the conservation law

$$
\frac{\partial C}{\partial t}=-\nabla \cdot C \mathbf{u}+\nabla \cdot(D \nabla C)-\nabla \cdot C \mathbf{u}_{\mathrm{T}},
$$

where $D$ is the Brownian diffusivity of the particles, $\mathbf{u}_{\mathrm{T}}$ is the thermophoretic velocity,

\footnotetext{
${ }^{2}$ For a complete list of symbols see Appendix B.
}

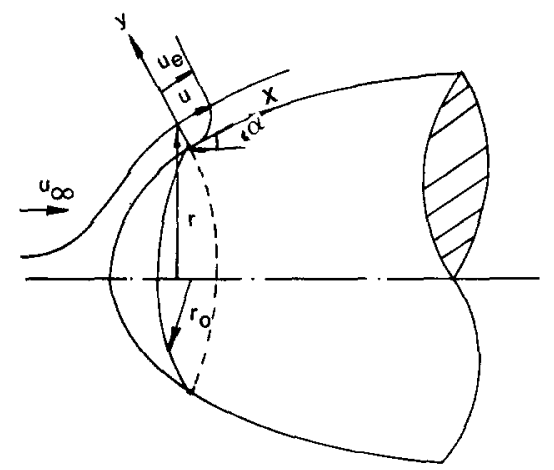

FIGURE 1

$$
\mathbf{u}_{\mathrm{T}}=-\frac{K \nu}{T} \operatorname{grad} T
$$

and $C$ is the number density of the particles. For compressible flow it is more convenient to introduce $\phi$, the particle concentration per unit mass of gas,

$$
\phi=C / \rho
$$

With $\phi$ as the unknown function and in the same boundary layer approximation the steady state form of [2.4] can be written as

$$
\begin{aligned}
\rho u \frac{\partial \phi}{\partial x}+\rho v \frac{\partial \phi}{\partial y}= & \frac{1}{r^{k}} \frac{\partial}{\partial y}\left[r^{k} D \frac{\partial \phi \rho}{\partial y}\right] \\
& -\frac{1}{r^{k}} \frac{\partial}{\partial y}\left(r^{k} \phi \rho u_{\Upsilon}\right)
\end{aligned}
$$

where $u_{\mathrm{T} y}$ is the component of $\mathbf{u}_{\mathrm{T}}$ normal to the surface. The particle diffusivity $D$ is much smaller than any of the gas diffusivities such as $\nu$. For example, for particles of diameter $0.05-1.0 \mu \mathrm{m}, D$ ranges from $2.4 \times 10^{-3}$ to 2.8 $\times 10^{-5} \mathrm{~m}^{2} / \mathrm{s}$ and the Schmidt number Sc $=\nu / D$ ranges from $6 \times 10^{3}$ to $5 \times 10^{5}$. So the effect of particle diffusion can be neglected except in the diffusion boundary layer which is expected to be very thin even in comparison with the boundary layer thickness. As in (1$3,5-7)$, the particle diffusion term on the right-hand side of [2.7] is neglected and [2.7] is reduced to 


$$
\begin{aligned}
\rho u \frac{\partial \phi}{\partial x}+\rho v \frac{\partial \phi}{\partial y} & \\
& =\frac{1}{r^{k}} \frac{\partial}{\partial y}\left(r^{k} \phi \rho \frac{K \nu}{T} \frac{\partial T}{\partial y}\right) .
\end{aligned}
$$

Accordingly specification of $\phi_{\infty}$ in the oncoming stream is sufficient for the determination of $\phi$. To find the rate of deposition at the surface it is not necessary to consider conditions inside the very thin concentration boundary layer because the gas velocity is extremely low there. The particles that enter this boundary layer by thermophoresis deposit nearly at the same streamwise position onto the wall.

After the $u, T$, and $\phi$ fields are determined by solving Eqs. [2.2], [2.3], and [2.8] the local deposition rate defined as the local flux of particles to the wall due to thermophoresis can be found easily,

$$
J=-C_{\mathrm{w}} \mathbf{n} \cdot \mathbf{u}_{\mathrm{Tw}}=\rho_{\mathrm{w}} \phi_{\mathrm{w}} \frac{K \nu}{T_{\mathrm{w}}}\left(\frac{\partial T}{\partial y}\right)_{\mathrm{w}},
$$

where $\phi_{\mathrm{w}}$ is the value of $\phi$ near the wall determined from [2.8] with diffusivity neglected.

3. SOLUTION FOR THE CONCENTRATION AND DEPOSITION RATE IN FLOWS NEAR THE STAGNATION POINT AND PAST A FLAT PLATE

In this section we give the solution for the particle concentration $\phi$ and deposition rate $J$ in the flow near the stagnation point on a cylinder or a sphere obtained by a procedure which invokes the Howarth-Dorodnitsyn transformation. The stagnation point case is investigated separately in this section in order to compare the results with those obtained for the same flow system but on the assumption of incompressibility (7) to show how the accounting of variable density affects the predicted values of particle concentration and deposition rate. The governing equations for flow past a flat plate have a similar form and are discussed here incidentally to show the identity of our approach with that of Goren (1).

To give the velocity boundary layer equations for the three flow systems a form similar to that in the incompressible case we introduce the Howarth-Dorodnitsyn variable $Y$,

$$
Y=\int_{0}^{y}\left(\rho / \rho_{\infty}\right) d y
$$

and the stream functions $\Psi$ and similarity variables $\eta_{1}$ as

(a) flat plate $\Psi=\left(\nu u_{\infty} x\right)^{1 / 2} f\left(\eta_{1}\right)$,

$$
\eta_{1}=\frac{1}{2}\left(\frac{u_{\infty}}{u x}\right)^{1 / 2} Y
$$

(b) cylinder $\Psi=\left(\beta_{1} \nu\right)^{1 / 2} x f\left(\eta_{1}\right)$,

$$
\eta_{1}=\left(\frac{\beta_{1}}{\nu}\right)^{1 / 2} Y
$$

(c) sphere $\Psi=\left(\frac{1}{2} \beta_{1} \nu\right)^{1 / 2} x^{2} f\left(\eta_{1}\right)$,

$$
\eta_{1}=\left(\frac{2 \beta_{1}}{\nu}\right)^{1 / 2} Y \text {. }
$$

With

$$
\begin{aligned}
& u=\frac{\rho_{\infty}}{\rho} \frac{\partial \Psi}{\partial y}, \\
& v=-\left.\frac{\rho_{\infty}}{\rho} \frac{\partial \Psi}{\partial x}\right|_{y},
\end{aligned}
$$

by using [3.1] and [3.2], Eqs. [2.2] and [2.3] can be transformed into

(a) flat plate $f^{\prime \prime \prime}+f f^{\prime \prime}=0, \quad f(0)=0$,

$$
f^{\prime}(0)=0, \quad f^{\prime \prime}(\infty)=2,
$$

(b) cylinder $f^{\prime \prime \prime}+f f^{\prime \prime}+\left(\frac{T}{T_{\infty}}-f^{\prime 2}\right)=0$,

$$
f(0)=0, \quad f^{\prime}(0)=0, \quad f^{\prime \prime}(\infty)=1 \text {, }
$$

(c) sphere $f^{\prime \prime \prime}+f f^{\prime \prime}+\frac{1}{2}\left(\frac{T}{T_{\infty}}-f^{\prime 2}\right)=0$,

$$
f(0)=0, f^{\prime}(0)=0, \quad f^{\prime \prime}(\infty)=1,
$$

and

$T^{\prime \prime}+\sigma f T^{\prime}=0, T(0)=T_{\mathrm{w}}, T(\infty)=T_{\infty}$,

for all three cases. In the derivation of [3.4] and [3.5] the assumptions $\mu \rho=\mu_{\infty} \rho_{\infty}$ and $M_{\infty}^{2} \ll 1$ are made. The former is a well-known 
simplification and is close to reality for not too large variations in temperature. The implication of the latter is that the speed of the oncoming gas is not too large, the range of which for the applicability of the procedure is further discussed in Section 6. The concentration Eq. [2.8] in the transformed coordinate system is written as

$\left[\frac{K T^{\prime}}{T}+f\right] \phi^{\prime}-\left[K\left(\frac{T^{\prime}}{T}\right)^{2}+K \sigma f \frac{T^{\prime}}{T}\right] \phi=0$.

With the boundary condition

$$
\phi(\infty)=\phi_{\infty},
$$

the solution of [3.6] can be written as

$$
\frac{\phi}{\phi_{\infty}}=\exp \left(-\int_{\eta_{1}}^{\infty} \frac{\left(T^{\prime} / T\right)^{2}-\left(T^{\prime \prime} / T\right)}{\left(T^{\prime} / T\right)+(f / K)} d \eta_{1}\right) .
$$

The Eqs. [3.4], [3.5], [3.8] for $f, T$, and $\phi$ have almost the same form as those for $f, T$, and $C$ in the case of incompressible fluid (see (7)). The only difference is in the momentum equations for the cylinder and sphere; instead of constant 1 there stands the ratio of temperatures $T / T_{\infty}$. Equations [3.4], [3.5] are solved by the Runge-Kutta method and the particle concentration $\phi$ is obtained from [3.8] by a quadrature of functions $f$ and $T$. For a flat plate our result is identical with that of Goren (1) as can be expected and will not be given here. The values of $\phi_{w} / \phi_{\infty}$ at the body surface for a cylinder and a sphere are given in Table I. One can see from the comparison of the data given here with those given in Table I of (7) that even for $T_{\mathrm{w}} / T_{\infty}$ much less than 1 the values of $\phi_{\mathrm{w}} / \phi_{\infty}$ are approximately the same as those of $C_{\mathrm{w}} / C_{\infty}$ obtained in the assumption of incompressible fluid. But the relationship between $\phi$ and $C$, i.e., Eq. [2.6], shows

$$
\frac{\phi_{\mathrm{w}}}{\phi_{\infty}}=\frac{C_{\mathrm{w}}}{C_{\infty}} \frac{T_{\mathrm{w}}}{T_{\infty}} .
$$

So the assumption of constant density would result in an incorrect reduction of the value of concentration by a factor of $T_{\mathrm{w}} / T_{\infty}$.

As for the local deposition rate, in the assumption of incompressibility one would have

$$
J_{\text {inc. }}=\left.\left(\frac{C_{\mathrm{w}}}{C_{\infty}}\right)_{\text {inc. }} C_{\infty} \frac{K \nu}{T_{\mathrm{w}}}\left(\frac{\partial T}{\partial y}\right)_{\text {inc. }}\right|_{y=0} .
$$

For compressible fluid one has

$$
J_{\mathrm{c} .}=\left.\left(\frac{\phi_{\mathrm{w}}}{\phi_{\infty}}\right)_{\mathrm{c} .} C_{\infty} \frac{\rho_{\mathrm{w}}}{\rho_{\infty}} \frac{K \nu}{T_{\mathrm{w}}}\left(\frac{\partial T}{\partial y}\right)_{\mathrm{c} .}\right|_{y=0} .
$$

\begin{tabular}{|c|c|c|c|c|c|c|}
\hline \multirow[b]{2}{*}{$H$} & \multicolumn{6}{|c|}{$T_{w} / T_{\infty}$} \\
\hline & 0.2 & 0.333 & 0.5 & 0.667 & 0.8 & 0.9 \\
\hline \multirow[t]{2}{*}{0.8} & 0.2198 & 0.3618 & 0.5336 & 0.6984 & 0.8247 & 0.9147 \\
\hline & 0.2195 & 0.3614 & 0.5332 & 0.6980 & 0.8241 & 0.9146 \\
\hline \multirow[t]{2}{*}{0.7} & 0.2320 & 0.3790 & 0.5534 & 0.7164 & 0.8377 & 0.9225 \\
\hline & 0.2316 & 0.3784 & 0.5526 & 0.7157 & 0.8372 & 0.9223 \\
\hline \multirow[t]{2}{*}{0.5} & 0.2640 & 0.4227 & 0.6015 & 0.7583 & 0.8675 & 0.9393 \\
\hline & 0.2631 & 0.4214 & 0.6000 & 0.7571 & 0.8667 & 0.9389 \\
\hline \multirow{2}{*}{0.3} & 0.3152 & 0.4887 & 0.6688 & 0.8124 & 0.9034 & 0.9582 \\
\hline & 0.3136 & 0.4865 & 0.6665 & 0.8106 & 0.9023 & 0.9578 \\
\hline \multirow{2}{*}{$\begin{array}{r}0.15 \text { (b) } \\
\text { (c) }\end{array}$} & 0.3881 & 0.5747 & 0.7474 & 0.8688 & 0.9375 & 0.9749 \\
\hline & 0.3854 & 0.5712 & 0.7442 & 0.8667 & 0.9363 & 0.9744 \\
\hline \multirow[t]{2}{*}{0.1} & 0.4317 & 0.6217 & 0.7861 & 0.8941 & 0.9516 & 0.9813 \\
\hline & 0.4284 & 0.6178 & 0.7827 & 0.8919 & 0.9504 & 0.9809 \\
\hline
\end{tabular}

\section{TABLE I}

Values of the Particle Concentration Ratio $\phi_{\mathrm{w}} / \phi_{\infty}$ at the Body Surface

Note. Lines (b) refer to the flow near the stagnation point on a cylinder, and (c) to that on a sphere. 
From [3.10] and [3.11] one concludes that

$$
J_{\text {inc. }}=\left(\frac{\rho_{\infty}}{\rho_{\mathrm{w}}}\right)^{2} J_{\mathrm{c} .}=\left(\frac{T_{\mathrm{w}}}{T_{\infty}}\right)^{2} J_{\mathrm{c} .},
$$

because

$$
\left(\frac{C_{\mathrm{w}}}{C_{\infty}}\right)_{\text {inc. }} \approx\left(\frac{\phi_{\mathrm{w}}}{\phi_{\infty}}\right)_{\mathrm{c} .}
$$

and

$$
\left.\left(\frac{\partial T}{\partial y}\right)_{c .}\right|_{y=0}=\left.\frac{\rho_{\mathrm{w}}}{\rho_{\infty}}\left(\frac{\partial T}{\partial y}\right)_{\text {inc. }}\right|_{y=0} .
$$

The latter relationship can be obtained from the similarity of the governing equations and boundary conditions for both flow systems and the Howarth-Dorodnitsyn transformation. So the assumption of constant density would reduce incorrectly the result for the local deposition rate by a factor of $\left(\rho_{\infty} / \rho_{\mathrm{w}}\right)^{2}$ or $\left(T_{\mathrm{w}} / T_{\infty}\right)^{2}$.

In this section the significance of the density variation effect in the cases of flow past a flat plate and near the stagnation point of a cylinder and a sphere was shown. But it is to be emphasized that the quantitative measure of this effect in each case must be analyzed concretely.

\section{METHOD OF SOLUTION IN THE GENERAL CASE}

To solve the boundary layer Eqs. [2.2], [2.3], [2.8] in the general case the ManglerLevy-Lees transformation (see (9-11)),

$$
\begin{aligned}
& d \xi=\rho_{\mathrm{e}} \mu_{\mathrm{e}} u_{\mathrm{e}}(\nu / a)^{2 k} d x, \\
& d \eta=\left[\rho u_{\mathrm{e}} /(2 \xi)^{1 / 2}\right](r / a)^{k} d y,
\end{aligned}
$$

is introduced, where $a$ is the characteristic length of the body. With $f^{\prime} \equiv u / u_{\mathrm{e}}, g \equiv I / I_{\mathrm{e}}$, and $w \equiv \phi / \phi_{\mathrm{e}}$ as unknown functions these equations can be written in the form

$$
\begin{aligned}
& \left(b f^{\prime \prime}\right)^{\prime}+f f^{\prime \prime}+\beta\left(c-\left(f^{\prime}\right)^{2}\right) \\
& =2 \xi\left(f^{\prime} \partial f^{\prime} / \partial \xi-f^{\prime \prime} \partial f / \partial \xi\right) \\
& \left(a_{1} g^{\prime}+a_{2} f^{\prime} f^{\prime \prime}\right)^{\prime}+f g^{\prime} \\
& =2 \xi\left(f^{\prime} \partial g / \partial \xi-g^{\prime} \partial f / \partial \xi\right)
\end{aligned}
$$

$$
\begin{aligned}
& \sigma K\left(a_{1}\left(g^{\prime} / g\right) w\right)^{\prime}+f w^{\prime} \\
& =2 \xi\left(f^{\prime} \partial w / \partial \xi-w^{\prime} \partial f / \partial \xi\right)
\end{aligned}
$$

where

$$
\begin{aligned}
b & =(1+t)^{2 k} \rho \mu / \rho_{\mathrm{e}} \mu_{\mathrm{e}}, \\
\beta & =\left(2 \xi / u_{\mathrm{e}}\right)\left(d u_{\mathrm{e}} / d \xi\right), \\
c & =\rho_{\mathrm{e}} / \rho, \\
a_{1} & =(1+t)^{2 k} \rho \mu /\left(\sigma \mu_{\mathrm{e}} \rho_{\mathrm{e}}\right), \\
a_{2} & =(1+t)^{2 k}(1-(1 / \sigma)) \rho \mu u_{\mathrm{e}}^{2} /\left(I_{\mathrm{e}} \rho_{\mathrm{e}} \mu_{\mathrm{e}}\right),
\end{aligned}
$$

and ' denotes the derivative with respect to $\eta$, and $t$ is the transverse curvature parameter, representing the deviation of $r$ from $r_{0}$ (see Fig. 1),

$$
\frac{r}{r_{0}}=1+\frac{y \cos \alpha}{r_{0}}=1+t
$$

The boundary conditions for [4.3]-[4.5] are

$$
\begin{aligned}
f^{\prime}(\xi, 0)=0, & \lim _{\eta \rightarrow \infty} f^{\prime}(\xi, \eta)=1, \\
g(\xi, 0)=0, & \lim _{\eta \rightarrow \infty} g(\xi, \eta)=1, \\
& \lim _{\eta \rightarrow \infty} w(\xi, \eta)=1 .
\end{aligned}
$$

The momentum and energy Eqs. [4.3], [4.4] are the usual laminar boundary layer equations and are solved in the present paper by the Keller's box method as described in Cebeci and Smith (11). The algorithm will not be repeated here except that a few corrected formulae needed are indicated in Appen$\operatorname{dix}$ A.

As for the particle concentration equation, we note that it is an equation of first order and its difference equation can be written as

$$
\begin{array}{r}
\frac{\sigma K\left[\left(a_{1}^{n}\left(G^{n} / g^{n}\right)\right)_{j} w_{j}^{n}-\left(a_{1}^{n}\left(G^{n} / g^{n}\right)\right)_{j-1} w_{j-1}^{n}\right]}{h_{j}} \\
+\frac{\left[\left(1+\alpha_{n}\right) f^{n}-\alpha_{n} f^{n-1}\right]_{j-1 / 2}\left(w_{j}^{n}-w_{j-1}^{n}\right)}{h_{j}} \\
-2\left(\alpha_{n} u^{n-1 / 2}\right)_{j-1 / 2} \frac{w_{j}^{n}+w_{j-1}^{n}}{2}=S_{j-1 / 2}^{n-1},
\end{array}
$$


where $G \equiv g^{\prime}$, and $h_{j}$ is the net spacing in the $\eta$ direction, $j=1,2, \ldots, J, \eta_{J}=\eta_{\infty}$.

$S_{j-1 / 2}^{n-1} \equiv$

$$
\begin{aligned}
& -\left\{\sigma K \left[\frac{\left(a_{1}^{n-1}\left(G^{n-1} / g^{n-1}\right)\right)_{j} w_{j}^{n-1}}{h_{j}}\right.\right. \\
& \left.-\frac{\left(a_{1}^{n-1}\left(G^{n-1} / g^{n-1}\right)\right)_{j-1} w_{j-1}^{n-1}}{h_{j}}\right] \\
& +f_{j-1 / 2}^{n-1} \frac{w_{j}^{n-1}-w_{j-1}^{n-1}}{h_{j}} \\
& +2 \alpha_{n}\left[u_{j-1 / 2}^{n-1 / 2} \frac{w_{j}^{n-1}+w_{j-1}^{n-1}}{2}\right. \\
& \left.\left.+\frac{1}{2}\left(f^{n}-f^{n-1}\right)_{j-1 / 2} \frac{w_{j}^{n-1}-w_{j-1}^{n-1}}{h_{j}}\right]\right\},
\end{aligned}
$$

with $\alpha_{n}=2 \xi_{n-1 / 2} / k_{\mathrm{n}}, k_{\mathrm{n}}$ being the net spacing in the $\xi$ direction, $n=1,2, \ldots, N$. Equation [4.12] can be solved by the recurrence formula

$$
w_{j-1}^{n}=\frac{S_{j-1 / 2}^{n-1}}{B_{j}}-\frac{A_{j} w_{j}^{n}}{B_{j}},
$$

where

$$
\begin{aligned}
& A_{j}=\frac{\sigma K}{h_{j}}\left(a_{1}^{n} \frac{G^{n}}{g^{n}}\right)_{j}+C_{j}-D_{j}, \\
& B_{j}=-\frac{\sigma K}{h_{j}}\left(a_{1}^{n} \frac{G^{n}}{g^{n}}\right)_{j-1}-C_{j}-D_{j}, \\
& C_{j}=\frac{\left[\left(1+\alpha_{n}\right) f^{n}-\alpha_{n} f^{n-1}\right]_{j-1 / 2}}{h_{j}}, \\
& D_{j}=\left(\alpha_{n} u^{n-1 / 2}\right)_{j-1 / 2} .
\end{aligned}
$$

The coefficients of Eq. [4.13] are all known functions after the convergent solutions of the difference equations of [4.3], [4.4] are obtained. With the same accuracy the functions $w_{J-1}, \ldots, w_{1}$ can be found from [4.13] successively with the boundary condition $w_{J}=1$. Then the formula [2.9] is used to calculate the deposition rate.

The system of [4.1]-[4.13] with [2.9] can be used to solve any external flow problem of thermophoretic deposition provided the body form and the outer flow parameters are given. We illustrate the numerical procedure on examples of thermophoretic deposition of particles in flow around a cylinder and sphere.

\section{EXAMPLES OF CALCULATIONS OF} THERMOPHORETIC DEPOSITION OF PARTICLES ON A CYLINDER AND A SPHERE

As examples of application of the method of the previous section we consider the flows of gas containing suspended particles around a cold cylinder and sphere. For the range of velocities usually encountered in thermophoretic problems the Mach number of the outer flow is very small: $M_{\mathrm{e}}^{2} \ll 1$. In that case

$$
g=\frac{I}{I_{\mathrm{e}}}=\frac{c_{\mathrm{p}} T+(1 / 2) u^{2}}{c_{\mathrm{p}} T_{\mathrm{e}}+(1 / 2) u_{\mathrm{e}}^{2}} \approx \frac{T}{T_{\mathrm{e}}},
$$

so that in Eq. [4.3]

$$
c=\frac{\rho_{\mathrm{e}}}{\rho}=\frac{T}{T_{\mathrm{e}}} \approx g .
$$

Equations [4.3], [4.4] can be further simplified when the well-known assumption

$$
\rho \mu=\rho_{\mathrm{e}} \mu_{\mathrm{e}}
$$

is made as was already done in Section 3. Finally, we neglect the transverse curvature effect in the flow past a sphere; that is, we make the assumption $t \approx 0$, which is valid approximately when $\operatorname{Re}_{\infty}$ is of the order $10^{4}$ or larger. Of course, the transverse curvature effect could be taken into account without any principal difficulty in which case the calculation would have to be made for each value of $\mathrm{Re}_{\infty}$ and the parameter $\operatorname{Re}_{\infty}$ would appear in the presentation of the results for a sphere.

For the flow past a circular cylinder we have the velocity of the inviscid, incompressible flow outside the boundary layer

$$
u_{\mathrm{e}}=2 u_{\infty} \sin \theta,
$$

where $\theta=x / a, a$ being the radius of the cylinder. From [4.1] and [4.6] we see

$\xi=-2 \rho \mu u_{\infty} a(\cos \theta-1)$, 


$$
\begin{aligned}
\beta & =\frac{\xi}{\rho \mu u_{\infty} a} \frac{\cos \theta}{\sin ^{2} \theta}=\frac{2 \cos \theta(1-\cos \theta)}{\sin ^{2} \theta}, \\
& =1, \quad \text { at the stagnation point. }
\end{aligned}
$$

For the flow past a sphere

$$
\begin{aligned}
u_{\mathrm{e}} & =\frac{3}{2} u_{\infty} \sin \theta \\
\xi & =\frac{3}{2} \rho \mu u_{\infty} a\left(\frac{1}{3} \cos ^{3} \theta-\cos \theta+\frac{2}{3}\right) \\
\beta & =\frac{4}{3} \frac{\xi}{\rho \mu u_{\infty} a} \frac{\cos \theta}{\sin ^{4} \theta} \\
& =2\left(\frac{1}{3} \cos ^{3} \theta-\cos \theta+\frac{2}{3}\right) \frac{\cos \theta}{\sin ^{4} \theta} \\
& =\frac{1}{2}, \quad \text { at the stagnation point. }
\end{aligned}
$$

With [5.2]-[5.9] substituted into Eqs. [4.3] and [4.4], they are solved by Keller's box method and then the concentration is obtained from [4.13] for a variety of parameters of $T_{w} /$ $T_{\infty}$ and $\sigma K$.

In the calculations, equal net spacing $h$ $=0.1$ in the normal direction was used. Along the tangential direction of the surface the dimensionless coordinate $\bar{\xi}=\xi / \rho \mu u_{\infty} a$ was introduced. The spacing of $\bar{\xi}$ was determined by uniform division of $\theta$ into equal intervals of $\pi / 20$ through formulae [5.5] and [5.8].

To test the accuracy of the method the solution at the stagnation point has been compared with the results obtained in Section 3. The solutions by the present method have been found to be very close to those of Section 3 as can be seen from the comparison of Table I with Table II where the data obtained by the present method are given. To economize space, only data for $\sigma K=0.5$ are presented. One can conclude that the algorithm in this section has the same accuracy as the RungeKutta method applied to the transformed equations according to Howarth and Dorodnitsyn.

In Fig. 2 the wall values $\phi_{\mathrm{w}} / \phi_{\infty}$ of concentration at the stagnation point of a cylinder for various $T_{\mathrm{w}} / T_{\infty}$ are shown as functions of $\sigma K$. The values for a sphere differ very little from those for a cylinder as is evident from Tables I and II.

The variation of the concentration along the surface can be calculated for each set of parameters of $\sigma K$ and $T_{\mathrm{w}} / T_{\infty}$. In Fig. 3 the distributions of $\phi_{\mathrm{w}} / \phi_{\infty}$ as functions of $\theta$ for $\sigma K$ $=0.576$ wit $T_{\mathrm{w}} / T_{\infty}=0.1$ and 0.5 are shown for a cylinder. It is noted that $\phi_{\mathrm{w}} / \phi_{\infty}$ remains almost constant along the surface and this is true for other values of $\sigma K$ and $T_{\mathrm{w}} / T_{\infty}$ and for a sphere.

The local deposition rate can be expressed as

$$
\begin{aligned}
J & =\rho_{\mathrm{w}} \phi_{\mathrm{w}} \frac{K \nu_{\mathrm{w}}}{T_{\mathrm{w}}}\left(\frac{\partial T}{\partial y}\right)_{\mathrm{w}} \\
& =K \frac{\rho_{\mathrm{w}} \mu_{\mathrm{w}} u_{\mathrm{e}}}{\sqrt{2}\left(\operatorname{Re}_{\infty}\right)^{1 / 2}(\bar{\xi})^{1 / 2}}\left(\frac{r}{a}\right)^{k}\left(\frac{1}{g^{1-\omega}} \frac{\partial g}{\partial \eta}\right)_{\mathrm{w}},
\end{aligned}
$$

\begin{tabular}{|c|c|c|c|c|c|c|}
\hline \multirow[b]{2}{*}{$H$} & \multicolumn{6}{|c|}{$T_{w} / T_{\infty}$} \\
\hline & 0.2 & 0.333 & 0.5 & 0.667 & 0.8 & 0.9 \\
\hline 0.5 (b) & 0.2640 & 0.4227 & 0.6014 & 0.7582 & 0.8674 & 0.9392 \\
\hline (c) & 0.2632 & 0.4214 & 0.6000 & 0.7570 & 0.8667 & 0.9389 \\
\hline
\end{tabular}

where $\omega$ is the power index in the viscosity law: $\mu_{\mathrm{w}} / \mu_{\infty}=\left(T_{\mathrm{w}} / T_{\infty}\right)^{\omega}=g_{\mathrm{w}}^{\omega}$. It is convenient to introduce the dimensionless deposition rate

$$
\bar{J} \equiv \frac{\sqrt{\operatorname{Re}_{\infty} J}}{C_{\infty} u_{\infty}} .
$$

TABLE II

Values of $\phi_{\mathrm{w}} / \phi_{\infty}$ at the Stagnation Point of a Cylinder and a Sphere Calculated by the Method Used in This Section 
For a cylinder we have

$$
\bar{J}_{\mathrm{C}}=2 K \frac{\phi_{\mathrm{w}}}{\phi_{\infty}} \frac{\sin \theta}{\sqrt{1-\cos \theta}}\left(\frac{1}{g_{\mathrm{w}}^{2-\omega}} \frac{\partial g}{\partial \eta}\right)_{\mathrm{w}}
$$

and at the stagnation point

$$
\bar{J}_{\mathrm{C}(\text { st.p. })}=2 \sqrt{2} K \frac{\phi_{\mathrm{w}}}{\phi_{\infty}}\left(\frac{1}{g^{2-\omega}} \frac{\partial g}{\partial \eta}\right)_{\mathrm{w}} .
$$

Analogously for a sphere we have

$\bar{J}_{\mathrm{S}}=\frac{3}{2} K \frac{\phi_{\mathrm{w}}}{\phi_{\infty}} \frac{\sin ^{2} \theta}{\sqrt{\cos ^{3} \theta-3 \cos \theta+2}}$

$$
\begin{array}{r}
\times\left(\frac{1}{g^{2-\omega}} \frac{\partial g}{\partial \eta}\right)_{\mathrm{w}}, \\
\bar{J}_{\text {S(st.p.) }}=\sqrt{3} K \frac{\phi_{\mathrm{w}}}{\phi_{\infty}}\left(\frac{1}{g^{2-\omega}} \frac{\partial g}{\partial \eta}\right)_{\mathrm{w}} .
\end{array}
$$

The dimensionless deposition rates at the stagnation point for a cylinder and a sphere for a variety of parameters of $\sigma K$ and $T_{\mathrm{w}} / T_{\infty}$ are calculated according to [5.12] and [5.14] after the solutions of [4.3], [4.4], [4.13] had been obtained by the procedure described above. They are shown in Figs. 4 and 5 as functions of $\sigma K$ with $T_{\mathrm{w}} / T_{\infty}$ as a parameter. The results can be used for a given set of parameters $C_{\infty}, u_{\infty}$, and $\mathrm{Re}_{\infty}$ to obtain the dimensional deposition rates from [5.10].

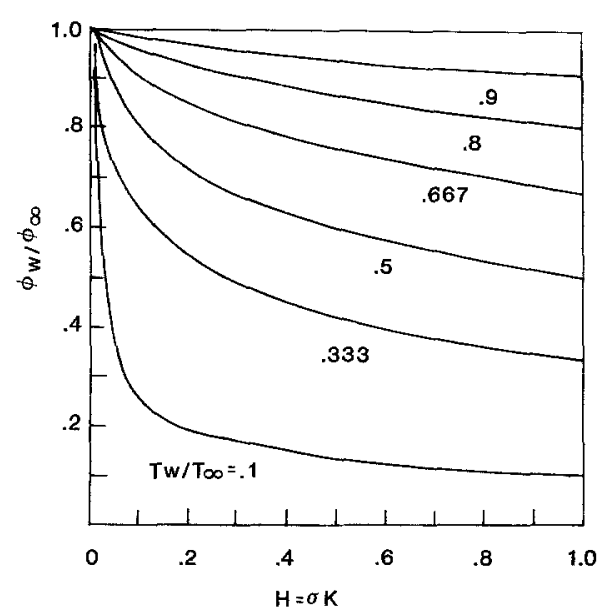

FIG. 2. $\phi_{\mathrm{w}} / \phi_{\infty}$ at the stagnation point of a cylinder.

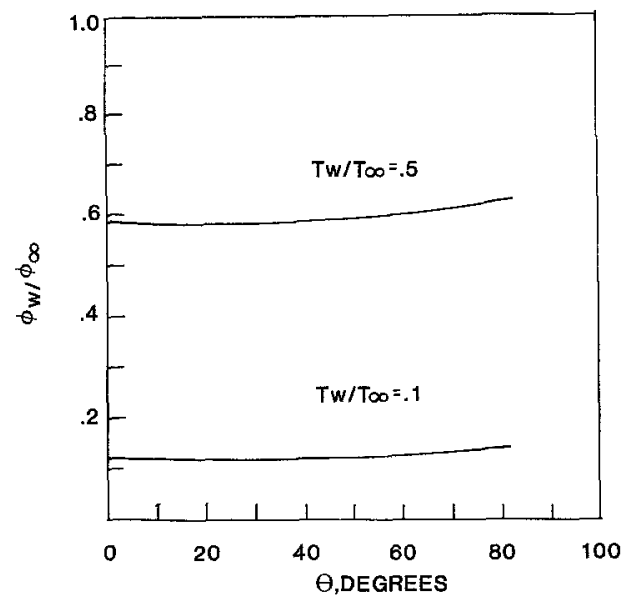

FIG. 3. $\phi_{w} / \phi_{\infty}$ along the surface of a cylinder for $\sigma K$ $=0.576, T_{\mathrm{w}} / T_{\infty}=0.1$ and 0.5 .

As for the deposition rate distribution along the surface it would seem to be necessary to calculate it each time for each set of parameters

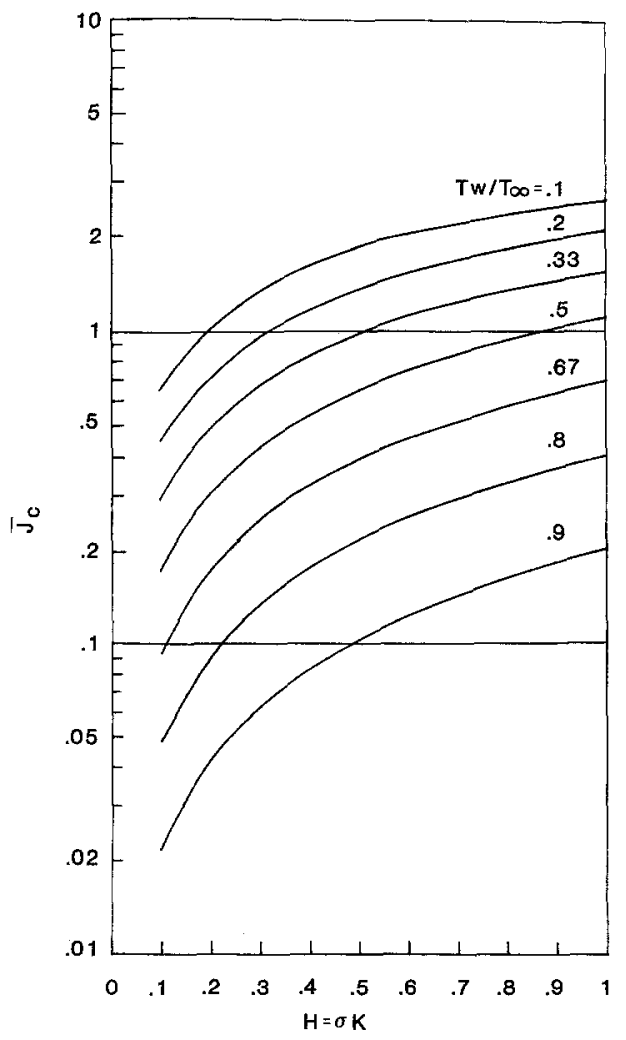

FIG. 4. Dimensionless deposition rate at the stagnation point of a cylinder.

Journal of Colloid and Interface Science, Vol. 127, No. 1, January 1989 


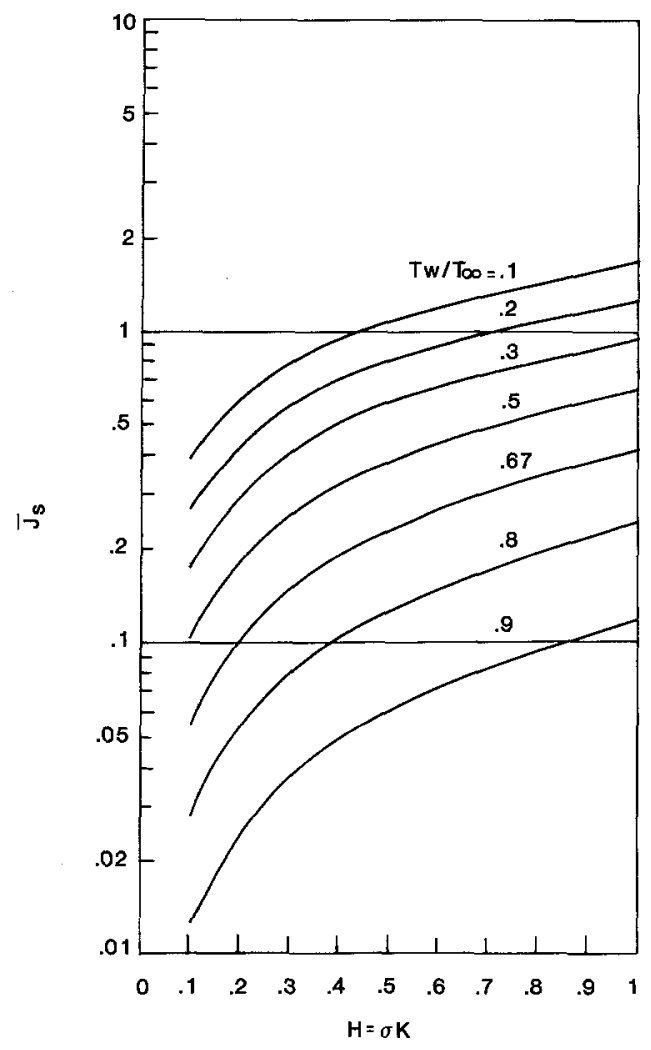

FIG. 5. Dimensionless deposition rate at the stagnation point of a sphere.

of $\sigma K$ and $T_{\mathrm{w}} / T_{\infty}$. As examples, the results for $\sigma K=0.576, T_{\mathrm{w}} / T_{\infty}=0.1$ and 0.5 for a cylinder and a sphere are shown in Figs. 6 and 7. It can be seen that although the absolute values of deposition rates differ rather greatly for $T_{\mathrm{w}} / T_{\infty}=0.1$ and $T_{\mathrm{w}} / T_{\infty}=0.5$, the relative distributions are almost identical. This is caused by the fact that the factor $\left(\phi_{\mathrm{w}} / \phi_{\infty}\right)(\partial g / \partial \eta)_{\mathrm{w}} / g_{\mathrm{w}}^{2-\omega}$ in formulae [5.11] and [5.13] remains almost constant when $\theta$ varies from zero to larger values downstream. As an example, the calculated values of the factor $K\left(\phi_{\mathrm{w}} / \phi_{\infty}\right)(\partial g / \partial \eta)_{\mathrm{w}} / g_{\mathrm{w}}^{2-\omega}$ for $K=0.8$, $T_{\mathrm{w}} / T_{\infty}=0.5$ as function of $\theta$ are shown to remain constant up to $\theta=72^{\circ}$ with an accuracy of $1 \%$. So for practical purposes for a variety of parameters of $\sigma K$ and $T_{\mathrm{w}} / T_{\infty}$ one can be sure that the distribution normalized by the stagnation point value has the form of $\sin \theta / \sqrt{2(1-\cos )}$ for a cylinder and of $\sqrt{3} \sin ^{2} \theta / 2\left(\cos ^{3} \theta-3 \cos \theta+2\right)^{1 / 2}$ for a sphere.

It is convenient to define the total dimensionless deposition rate as

$$
\bar{J}_{(\text {total })}=\frac{1}{S} \int_{S} \bar{J} d S,
$$

where $S$ is part of the body surface wetted by the oncoming flow. For a cylinder and a sphere it is well known that the real external flow field differs from the potential flow pattern given by [5.4] and [5.8] rather significantly owing to the separation of flow. The results presented are valid approximately up to $\theta=60^{\circ}-70^{\circ}$. If we suppose that formulae [5.11]-[5.13] are valid approximately for the front part of the surface and that the deposition at the rear part is zero, the total deposition rates for a cylinder and a sphere can be written approximately as

$$
\begin{aligned}
\bar{J}_{\mathrm{C}(\text { total })} & \approx \frac{2}{\pi a} \int_{0}^{\pi / 2} \bar{J}_{\mathrm{C}} a d \theta \\
& \approx \frac{2 \bar{J}_{\mathrm{C}(\text { st.p. })}}{\pi} \int_{0}^{\pi / 2} \frac{\sin \theta d \theta}{\sqrt{2(1-\cos \theta)}} \\
& \approx 0.9 \bar{J}_{\mathrm{C}(\text { st.p. })}
\end{aligned}
$$

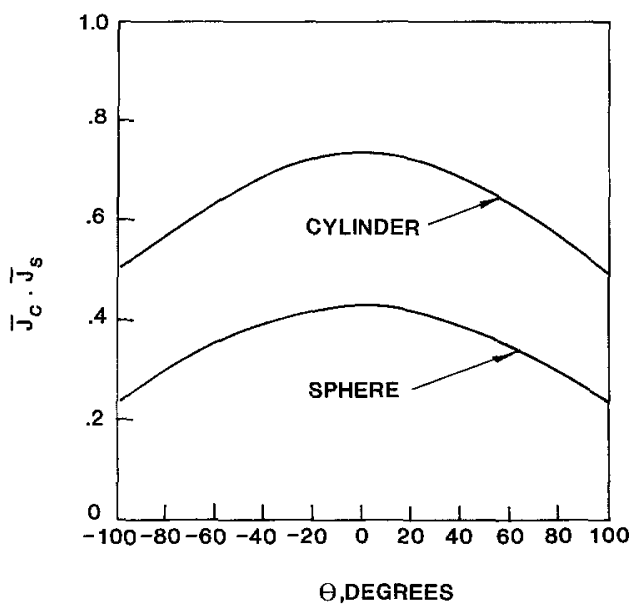

FIG. 6. Dimensionless deposition rate along the surface of a cylinder and a sphere. $T_{\mathrm{w}} / T_{\infty}=0.1, K=0.8$, and $\sigma$ $=0.72$. 


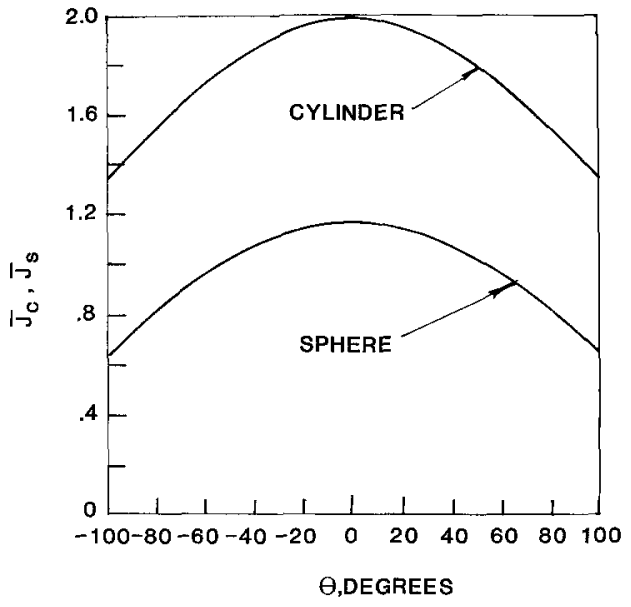

FIG. 7. Dimensionless deposition rate along the surface of a cylinder and a sphere. $T_{\mathrm{w}} / T_{\infty}=0.5, K=0.8$, and $\sigma$ $=0.72$.

$$
\begin{aligned}
\bar{J}_{\mathrm{S}(\text { total })} & \approx \frac{1}{2 \pi a^{2}} \int_{0}^{\pi / 2} \bar{J}_{\mathrm{S}} 2 \pi a^{2} \sin \theta d \theta \\
& \approx \bar{J}_{\mathrm{S}(\text { st.p. })} \int_{0}^{\pi / 2} \frac{(\sqrt{3} / 2) \sin ^{3} \theta d \theta}{\sqrt{\cos ^{3} \theta-3 \cos \theta+2}} \\
& \approx 0.817 \bar{J}_{\mathrm{S}(\text { st.p. }) .}
\end{aligned}
$$

So one can turn to Figs. 4 and 5 for the dependence of $\bar{J}_{\mathrm{C}(\text { total })}$ and $\bar{J}_{\mathrm{S}(\text { total })}$ on parameters $\sigma K$ and $T_{\mathrm{w}} / T_{\infty}$.

It is interesting to compare the results of the present approach with those obtained from const. density considerations. We note first that the dimensionless local deposition flux used in (5) (designated further in this paper as $\left.\bar{J}_{(5)}\right)$ was defined there implicitly as (in notation of (5))

$$
\bar{J}_{(5)}=\frac{\sqrt{\operatorname{Re}} J}{C_{\infty} u_{1} R} .
$$

For a cylinder from (5) we have $u_{1} R=u_{\infty}$, $\operatorname{Re}=2 u_{\infty} a / \nu$, but it was not specified under what temperature $\nu$ ought to be measured. If we suppose $\nu$ is determined at the bottom of the boundary layer one has the relationship between $\bar{J}_{(5)}$ and our $\bar{J}_{\mathrm{C}}$

$$
\bar{J}_{(5)}=\frac{\sqrt{2 \mathrm{Re}_{\mathrm{w}} J}}{C_{\infty} \cdot 2 u_{\infty}}=\frac{1}{\sqrt{2}}\left(\frac{1}{g_{\mathrm{w}}}\right)^{(1+\omega) / 2} \bar{J}_{\mathrm{C}} \text {. }
$$

From Table I and Fig. 2 of (5) one has the local particle flux at the stagnation point in the incompressible assumption for $g_{\mathrm{w}}=0.5$ and $K=0.8, \sigma=0.71$

$$
\bar{J}_{(5)}=0.233 \text {, }
$$

or in our notation ( $g_{\mathrm{w}}=0.5, \omega=0.77$ is supposed)

$$
\bar{J}_{\mathrm{C} \text { (st.p.)inc. }}=0.178
$$

while from our calculation for the same set of parameters (except for a small difference in $\sigma$, $\sigma=0.72$ in our case) one has (see Figs. 4 and 6)

$$
\bar{J}_{\text {C(st.p.). }}=0.732 \text {. }
$$

By comparing [5.20] and [5.21] we see that

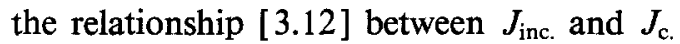
holds within the accuracy of calculation. As for the distribution of the deposition rate we note that the distribution form of $\bar{J}_{C}$ is quite different from that obtained in the incompressible assumption (cf. (5, Fig. 2)). As there is no similarity transformation from compressible into incompressible flow fields in the whole region of the flow, the explicit relationship between $\bar{J}_{\text {c. }}$ and $\bar{J}_{\text {inc. }}$ like that of the form [3.12] is not expected to exist in the general case.

\section{DISCUSSION}

In this paper our attention is focused on providing a general method of calculation of the thermophoretic deposition in external flow past bodies and on elucidating the significant density variation effect in thermophoretic problems of external flow. The predicted values of the wall concentration and deposition rate by the const. density assumption are shown to differ from the correct values by factors of $T_{\mathrm{w}} / T_{\infty}$ and $\left(T_{\mathrm{w}} / T_{\infty}\right)^{2}$, respectively, for flows near the stagnation point of a cylinder and a sphere and a past flat plate. But it is emphasized that this is not a universal conclusion. Numerical examples are given for flows around a cylinder and a sphere, the procedure being quite common in solving prob- 
lems of flows past bodies of arbitrary shape. It has been mentioned that the potential flow patterns given by [5.4] and [5.8] differ from the real flow outside the boundary layer because of flow separation. When the external flow field $U(x)$ is known either from theoretical consideration or from experimental investigations the numerical algorithm can be carried out in the same way to get more realistic results.

The method used in this paper has direct applications in problems like the staining of the surface of heat exchangers and the erosion of turbine blades by small particles sedimented on them. The analysis shows that these problems are more serious than they would seem to be from the constant density analysis.

For the validity of the boundary layer approximation the oncoming $\operatorname{Re}_{\infty}$ must be greater than $10^{3}$. For a characteristic length $a$ $\sim 0.1 \mathrm{~m}$, this implies that $u_{\infty}$ must be greater than $0.1 \mathrm{~m} / \mathrm{s}$. Greater $\operatorname{Re}_{\infty}$ is required to verify the neglect the transverse curvature effect in the axisymmetric flow case. This implies $u_{\infty}$ $>1 \mathrm{~m} / \mathrm{s}$ (for $a=0.1 \mathrm{~m})$. Furthermore, in the previous formulation it has been assumed that the particles follow the gas motion closely when thermophoresis is absent. This can be verified when the Stokes number $\mathrm{St}$ $=(2 / 9)\left(a_{\mathrm{p}} / a\right)^{2}\left(\rho_{\mathrm{p}} / \rho_{\infty}\right) \operatorname{Re}_{\infty}$, i.e., the ratio of the relaxation time of the particle due to viscosity to the gas flow characteristic time, is sufficiently small. For $a \sim 0.1 \mathrm{~m}$ and $a_{\mathrm{p}} \sim 1$ $\mu \mathrm{m}, \operatorname{Re}_{\infty}<10^{7}$ is implied. For smaller bodies smaller $\operatorname{Re}_{\infty}$ is required.

\section{APPENDIX A}

In the formula [7.9.11b] of (11) the correct expression for matrix $A$ for $j=J$ should have the special form (the notation of (11) is used)

$$
A_{J} \equiv\left[\begin{array}{cc}
-h_{J} / 2 & -h_{J} / 2 \\
\bar{\phi}_{J} & -\phi_{J}
\end{array}\right]
$$

instead of the general form indicated in (11). Analogously the $\alpha_{j}$ matrix in [7.9.17] for $j \mathrm{St}$ $=J$ also should have the special form

$$
\alpha_{J}=\left[\begin{array}{cc}
-h_{J} / 2+e_{J-1} & -h_{J} / 2 \\
-\bar{\phi}_{J}+\bar{\theta}_{J} e_{J-1} & \phi_{J}
\end{array}\right] .
$$

As a consequence, the following expressions for $x_{J}, y_{J}$,

$$
\begin{aligned}
& x_{J}=\left|\begin{array}{cc}
\mathrm{m}_{J} & -h_{J} / 2 \\
\mathrm{n}_{J} & \phi_{J}
\end{array}\right| /\left|\begin{array}{cc}
\left(\alpha_{11}\right)_{J} & -h_{J} / 2 \\
\left(\alpha_{21}\right)_{J} & \phi_{J}
\end{array}\right|, \\
& y_{J}=\left(2 / h_{J}\right)\left[\left(\alpha_{11}\right)_{J} x_{J}-\mathrm{m}_{J}\right],
\end{aligned}
$$

should replace the general formulae given in [7.9.23] of (11).

\section{APPENDIX B: NOMENCLATURE}

\section{Symbols}

a radius

$a_{1}$ cf. [4.6]

$a_{2}$ cf. [4.6]

$b \quad$ cf. [4.6]

c $\quad \rho_{\mathrm{e}} / \rho$

$C \quad \phi \rho$, number density of particles

$c_{\mathrm{p}} \quad$ specific heat

$D \quad$ Brownian diffusivity of particles

$f^{\prime} \quad u / u_{\mathrm{e}}$

$g \quad I / I_{\mathrm{e}}$

$G \quad g^{\prime}$

$H \quad \sigma K$, thermophoretic coefficient

$h_{j} \quad$ net spacing in $\eta$ direction

$I \quad c_{\mathrm{p}} T+u^{2} / 2$, total enthalpy

$J \quad$ local deposition rate, [2.9]

$\bar{J} \quad \sqrt{\operatorname{Re}_{\infty}} J /\left(C_{\infty} u_{\infty}\right)$, dimensionless deposition rate

$k \quad 1$, for axisymmetric flow

$k \quad 0$, for two-dimensional flow

$K$ thermophoretic coefficient

$k_{\mathrm{g}} \quad$ thermal conductivity of gas

$k_{\mathrm{n}} \quad$ net spacing in $\xi$ direction

$p \quad$ pressure

$r$ radial distance

$r_{0}$ radial distance from the body

$\operatorname{Re}_{\infty} \quad u_{\infty} a / \nu_{\infty}$, Reynolds number

$\operatorname{Re}_{\mathrm{w}} \quad u_{\infty} a / \nu_{\mathrm{w}}$, Reynolds number

$S \quad$ part of surface wetted by the oncoming flow

$\nu / \mathrm{D}$, Schmidt number

$S_{c}$
St

$(2 / 9)\left(a_{\mathrm{p}} / a\right)^{2}\left(\rho_{\mathrm{p}} / \rho_{\infty}\right) \operatorname{Re}_{\infty}$, Stokes number 
$t \quad y \cos \alpha / r_{0}$

$T$ temperature

$u, v \quad x, y$ components of the gas velocity

$\mathbf{u}_{\mathrm{T}}$ thermophoretic velocity, [2.5]

$w \quad \phi / \phi_{\mathrm{e}}$

$x, y$ coordinates along and normal to the surface

$Y \quad$ Howarth-Dorodnitsyn variable, [3.1]

$\alpha$

the body

$\beta \quad 2 \xi\left(d u_{\mathrm{e}} / d \xi\right) / u_{\mathrm{e}},[4.6]$

$\beta_{1} \quad$ outer edge speed parameter, [3.2]

$\eta \quad$ transformed $y$ coordinate, [4.2]

$\eta_{1} \quad$ similarity coordinate for stagnational case, [3.2]

$\theta \quad x / a$

$\mu \quad$ viscosity of the gas

$\nu \quad \mu / \rho$, kinematic viscosity

$\rho$ density

$\xi \quad$ transformed $x$ coordinate, [4.2]

$\bar{\xi} \quad \xi /\left(\rho_{\infty} \mu_{\infty} u_{\infty} a\right),[4.1]$

$\sigma \quad \mu c_{\mathrm{p}} / k_{\mathrm{g}}$

$\phi \quad C / \rho$, particle concentration per unit mass of gas

$\Psi \quad$ stream function

$\omega \quad$ power index of the viscosity law

\section{Indices}

c. compressible, variable density

C cylinder

e at the edge of boundary layer

g gas property

inc. incompressible, const. density $j \quad$ value at the $j$ th node point in $\eta$ direction

$n \quad$ value at the $n$th node point in $\xi$ direction

p particle property

S sphere

st.p. stagnation point value

w wall value

$\infty$ free stream value

\section{ACKNOWLEDGMENTS}

The author expresses his thanks to the National Natural Science Foundation of China for supporting this work. $\mathrm{He}$ also expresses his gratefulness to Professor G. K. Batchelor for his care and cardinal suggestions during this work.

\section{REFERENCES}

1. Goren, S. L., J. Colloid Interface Sci. 61, 77 (1977).

2. Walker, K. L., Homsy, G. M., and Geyling, F. T., J. Colloid Interface Sci. 69, 138 (1979).

3. Walker, K. L., Geyling, F. T., and Nagel, S. R., J. Amer. Ceram. Soc. 63, 552 (1980).

4. Talbot, L., Cheng, R. K., Schefer, R. W., and Willis, D. R., J. Fluid Mech. 101, 737 (1980).

5. Homsy, G. M., Geyling, H. T., and Walker, K. L., J. Colloid Interface Sci. 83, 495 (1981).

6. Weinberg, M. C., J. Amer. Ceram. Soc. 65, 81 (1982).

7. Batchelor, G. K., and Shen, C., J. Colloid Interface Sci. 107, 21 (1985).

8. Abbott, J. S., paper presented at SIAM meeting, Boston, July 1986.

9. Probstein, R. F., and Elliott, D., J. Aeronaut. Sci. 23, 208 (1956).

10. Hayes, W. D., and Probstein, R. F., "Hypersonic Flow Theory." Academic Press, New York, 1959.

11. Cebeci, T., and Smith, A. M. O., "Analysis of Turbulent Boundary Layers." Academic Press, New York, 1974. 